\title{
Estudio psicosocial sobre las representaciones sociales de género*
}

\section{A Psychosocial Study about the Social Representations of Gender}

Teresa Cristina Bruel dos Santos

Centro de Referência para Mulheres Vítimas de Violência Patrícia Esber, Brasil

Helena Beatriz K. Scarparo"* Pontifícia Universidade Católica do Rio Grande do Sul, Brasil

Aline Reis Calvo Hernandez Universidade Estadual do Rio Grande do Sul, Brasil

Julia Sebastián Herranz Universidad Autónoma de Madrid, España

Amalio Blanco Universidad Autónoma de Madrid, España

Recibido: 7 de diciembre de 2012 Revisado: 5 de marzo de 2013 Aceptado: 3 de abril de 2013

\section{Resumen}

Se presenta un estudio de las representaciones sociales acerca de lo masculino y lo femenino elaboradas por una muestra de 435 estudiantes de la Comunidad Autónoma de Madrid. Para el análisis de dichas representaciones se ha utilizado el método de Asociación Libre de Palabras a un conjunto de estímulos inductores. Los resultados del escalamiento multidimensional que asocia los siete estímulos inductores muestran dos claros campos semánticos: el primero, formado por los estímulos inductores desigualdad y violencia; el segundo, formado por los estímulos inductores hombre, masculino, mujer, femenino. La formación del campo semántico indica similitud, una relación de proximidad entre los términos asociados. Los análisis y la discusión de los hallazgos se centran en torno a las representaciones sociales de lo femenino y lo masculino, eje central del debate propuesto.

Palabras clave: psicosocial, representaciones sociales, género.

\section{Abstract}

The present study is about social representations of masculine and feminine held by a sample of 435 students from the Autonomous Community of Madrid.

* Artículo de investigación.

Correspondencia: Helena Beatriz K. Scarparo, Pontifícia Universidade Católica do Rio Grande do Sul, Brasil. Dirección postal: Av. Ipiranga, 6681 prédio 11, sala 929 - Porto Alegre - Brasil CEP - 90619900. Correo electrónico: scarparo@pucrs.br 
The analysis of the representations was made through a Free Word Association method to a number of inducing stimuli. The results of a multidimensional scaling that associates the seven inducing stimuli clearly show two semantic fields: the first includes inequality and violence as inducing stimuli, and the second is formed by man, masculine, woman, feminine as stimuli. The formation of the semantic field indicated similarity, a relationship of proximity among the associated terms. The analysis and the discussion of the findings are constructed around the social representations of what is masculine and feminine.

Keywords: psychosocial study, social representations, gender.

Las representaciones, los conocimientos sociales, expresan un deseo de aprender, de comprender, de tornar conocido lo no familiar. Se entienden las representaciones como entidades en sí mismas, realidades mentales; es decir, construcciones psíquicas y materiales: son informaciones acerca del conocimiento del "sentido común" (Moscovici, 1981). Al asumir que es imposible conocer sin representar, se está asumiendo que esas representaciones implican dimensiones simbólicas y sociales, se tornan fenómenos subjetivos, intersubjetivos y objetivos (Jovchelovitch, 2007).

Las experiencias, las informaciones y los conocimientos que se trasmiten a través de la educación y la comunicación son determinantes en la creación de las representaciones sociales, pues juegan un papel elemental en la construcción de consensos formulados en el ámbito de las relaciones de influencia y de pertenencia social. Las representaciones sociales (RS) son elaboradas a partir de la cultura de los grupos y de los procesos sociales de construcción de conocimiento (Moscovici, 1998). Las RS circulan y se cristalizan en los discursos, así como en las prácticas cotidianas, forman identidades e historias de los grupos y deben ser entendidas como una teoría acerca de cómo nuevos saberes son acomodados en el tejido social.

El concepto de RS, comprendido con una perspectiva psicosociológica, remite a una categoría del pensamiento a través de la cual grupos sociales elaboran, interpretan, expresan y organizan su realidad. De hecho, el eje central de la teoría de las representaciones sociales (TRS) parte del supuesto según el cual detrás de las acciones individuales reside una representación del mundo, una representación que trasciende el ámbito individual y pasa al social. La representación del mundo no es tan solo racional, cognitiva y lógica: es también una representación creada, fomentada y compartida socialmente, es todo un sistema circular de creencias y valores que no están encerrados dentro de un marco individual (Moscovici, 2003).

La TRS constituye uno de los marcos que guiaron la aproximación del grupo a cargo del presente trabajo al estudio de las percepciones y las creencias juveniles acerca de la categoría género. Dicho referente aporta numerosas herramientas epistemológicas y hermenéuticas para el entendimiento de este fenómeno: la interacción humana que se verifica entre los individuos y los grupos presupone la existencia de una representación; a la psicología social le corresponde analizar dichas interacciones, y entonces se asume que el estudio de las representaciones debe ser puesto en el centro de su interés; las RS son estructuras simbólicas originadas en la capacidad creativa del psiquismo humano; dicha teoría pretende dar cuenta de las mediaciones entre la vida individual y social (Guareschi y Jovchelovitch, 1995).

Se asume que esas representaciones del mundo son una construcción activa de los grupos, lo cual posibilita comprender que también los objetos puedan ser producidos por las representaciones del mundo. En ese sentido, se puede afirmar que solo existen realidades representadas. Quizá, lo más importante sea pensar que la representación del otro, de la interacción, activa un proceso de categorización tendiente a asignar una serie de cogniciones a determinado grupo, que pueden llegar a ser estereotipadas en la medida en que se asignan 
unas características permanentes a los miembros de una categoría. Tales cogniciones vinculadas a un determinado grupo sirven para prescribir conductas sociales. Estas últimas son, en general, automáticas y secuenciales, y siempre estarán reguladas por las representaciones sociales.

Representar es estar implicado en un sistema previo de creencias e imágenes generadas a partir de un conocimiento anterior que circula en el entorno y pasa a constituir, a través de la comunicación, comportamientos y acciones en ambientes concretos. Para Moscovici (1976, p. 17-18), la RS es

...una modalidad particular del conocimiento, cuya función es la elaboración de los comportamientos y la comunicación entre individuos. La representación es un corpus organizado de conocimientos y una de las actividades psíquicas gracias a las cuales los hombres hacen inteligible la realidad física y social, se integran en un grupo o en una relación cotidiana de intercambios.

Las RS expresan, eminentemente, el espacio del sujeto en su relación con la alteridad, en medio de su lucha para interpretar, entender y construir el mundo en el que vive. Al tiempo, las representaciones ayudan a los individuos a orientarse en su universo social y material.

Así, dicha teoría sirve para pensar sobre conjuntos de conceptos, proposiciones y explicaciones que se originan en las interacciones cotidianas y en las comunicaciones interpersonales. Las RS aluden a realidades compartidas por un grupo; también actúan como elemento explicativo y evaluativo, de tal modo que pueden considerarse la versión contemporánea del sentido común. Además, las RS constituyen modelos explicativos que le permiten a un grupo interpretar las experiencias propias y de los demás (Moscovici, 1987; Jodelet, 1985).

En el campo de las RS existe un terreno común de significados históricamente compartidos, porciones de sabiduría, memoria histórica, tradición y sentido común. Ellos presentan, en sus fundamentos, un núcleo estable y permanente, sostenido por los pilares de la cultura y la memoria de los grupos. Las RS de un grupo son clave para entender la estructura y el contenido sobre diferentes objetos reconocidos socialmente. Considerando que las RS incluyen visiones estereotípicas acerca de dichos objetos, el presente estudio permite poner de relieve el conjunto de principios organizadores y posiciones individuales y grupales relacionados con el saber común sobre las diferencias entre los géneros. A través de un análisis de las RS es posible entender las construcciones insertadas en la estructura social y que interfieren de lleno en las prácticas culturales.

Los estudios de género revelan que las representaciones se hallan íntimamente vinculadas a construcciones socioculturales que determinan papeles, características y afectos específicos para cada sexo. Esas prescripciones sociales se difunden a través de los procesos de socialización/comunicación, y así construyen identidades y patrones de comportamiento. Las definiciones sociales de femenino y masculino, como la definición de los patrones de comportamiento considerados propios, no se limitan a establecer una diferenciación binaria entre dichas categorías, sino que establecen diferencias asimétricas concretas, y así orientan creencias y actitudes. Se podría pensar que el sistema de creencias y representaciones cumple funciones específicas en los grupos sociales conforme sus intereses, sus posiciones políticas, sus pensamientos religiosos, entre otros.

Se busca, a través del presente estudio, situar el hecho de que las diferencias entre femenino y masculino se instalan dentro de una lógica intersubjetiva en los grupos sociales y se desarrollan de acuerdo con un modelo de transmisión de valores que determina y mantiene un orden hegemónico y asimétrico de poder que se propaga a través de la historia. Se trata, además, de considerar las representaciones un sistema interpretativo para los miembros de un determinado grupo que induce procesos de clasificación social. La importancia del estudio de las RS de género radica en hacer visibles las creencias, los valores, los supuestos ideológicos establecidos, con base en las diferencias biológicas, la adscripción diferenciada de características y los papeles sociales que sitúan a hombres y mujeres en posiciones dis- 
tintas. El concepto "género" pone de manifiesto la relación desigual entre mujeres y hombres en cuanto a sujetos sociales.

Dichos procesos son determinantes a la hora de delimitar creencias, imágenes, sentimientos y comportamientos (Pérez, Moscovici y Chulvi, 2002). Se puede decir que ciertas actitudes y comportamientos que priman por el predominio de un sexo sobre el otro han sido tradicionalmente justificados y adaptados en los procesos de socialización en las más variadas culturas, de forma que han obstaculizado el desarrollo de un sistema de valores igualitarios entre ambos sexos (Lorente Acosta, 2001; Amorós, 1990).

A través de un estudio exploratorio-descriptivo, en el presente trabajo se buscó identificar las estructuras, los significados y los conocimientos elaborados en las RS juveniles acerca de las diferencias de género. Por ello, el presente estudio tuvo como objetivo general conocer las percepciones, las atribuciones y las representaciones de género en los jóvenes españoles. Básicamente analizar las RS que los jóvenes formulan acerca de las diferencias de género; específicamente, sobre el posicionamiento acerca de las características atribuidas a lo femenino y lo masculino. La pertinencia de la presente investigación se plasma a través de la necesidad de conocer cómo piensan los jóvenes y como herramienta útil a la hora de plantear un quehacer futuro en términos de prevención e intervención; sobre todo, en el terreno de las políticas de igualdad de oportunidades ${ }^{1}$ y de una coeducación teñida de la perspectiva de género.

\section{Métodos}

\section{Participantes}

Se accedió a una muestra de conveniencia integrada por 435 estudiantes del $1^{\circ}, 2^{\circ}, 3^{\circ}$ de la Educación Secundaria Obligatoria (ESO) y Bachillerato de institutos de la Comunidad Autónoma

1 Pretenden la equiparación en derechos de quienes viven en situación de desigualdad. de Madrid²: 227 hombres y 208 mujeres, con una edad $M=14,87(D E 1,15)$. Dada la relevancia de la variable sexo en la interpretación de los resultados, se tuvo como criterio obtener un número equilibrado de mujeres y hombres.

\section{Instrumentos, procedimiento y análisis de la información}

En sesiones grupales, cuya duración osciló entre 45 y 60 minutos, los jóvenes participantes respondieron al instrumento de Asociación Libre de Palabras (Doise, Clémence y Lorenzi-Cioldi, 1993). El instrumento aplicado estaba compuesto por 7 términos que sirvieron de estímulos inductores. Los estímulos utilizados fueron: mujer, hombre, femenino, masculino, igualdad entre hombres y mujeres, desigualdad entre hombres y mujeres, y violencia contra las mujeres.

Durante la aplicación del instrumento se les informó a los participantes que recibirían una hoja con una serie de términos (i. e., o estímulos inductores), donde la tarea que ellos debían realizar sería escribir todas las palabras que les vinieran a la mente (sustantivos, adjetivos, etc.) asociadas al término presentado. Se les indicó a los participantes que no existían palabras incorrectas o correctas, y que el número de palabras asociadas era ilimitado, por lo cual podían escribir cuantas palabras desearan. Los estímulos inductores fueron presentados consecutivamente, en el mismo orden.

El método de Asociación Libre de Palabras posibilita elaborar diccionarios de palabras que reflejan el campo semántico elaborado en torno a cada estímulo. En su conjunto, los términos permiten observar cómo se posiciona la muestra respecto a los conceptos estudiados; es decir, un diccionario de palabras (Di Giacomo, 1980) contiene los significados asociados a cada término. Cabe señalar que se creó un diccionario para realizar análisis posteriores.

2 Nombres de los institutos: Instituto de Enseñanza Secundaria Soto del Real; Instituto de Enseñanza Iturralde; Colegio Santa María de los Rosales; Instituto de Enseñanza Secundaria Rosa Chacel; Instituto de Enseñanza Secundaria Sierra de Guadarrama. 
Para la elaboración del diccionario se utilizó un procedimiento relacionado directamente con las clásicas reglas del Análisis de Contenido: a) Todas las palabras son reducidas al masculino singular (por ejemplo: belleza-bello); b) Distintas formas de la misma palabra son reducidas a la forma más común en el diccionario (por ejemplo: bellezaguapo, por bello); c) Los adverbios y los sustantivos son reducidos a su correspondiente adjetivo, si este ya existe en el diccionario (por ejemplo: belleza por bello); d) Las combinaciones de palabras son reducidas al correspondiente adjetivo, cuando este exista en el índice; las combinaciones a las que no correspondan adjetivos son mantenidas (por ejemplo: afecto, afectiva, por sensibilidad); e) Las jergas son reducidas al adjetivo más cercano posible (por ejemplo: ligar por sexo); f) Algunas palabras que tienen el mismo sentido son reducidas a la palabra más común de la muestra, excepto cuando tenga varios sentidos posibles (por ejemplo: tiendas, maquillaje, ropas, bolso, por moda). De los siete estímulos estudiados, se optó por analizar dos estímulos, que fueron clave al estudio: femenino y masculino.

\section{Resultados}

Se hizo un análisis de la Asociación Libre de Palabras, centrado inicialmente en los resultados obtenidos mediante el escalamiento multidimensional (MDS), técnica que aglutina y representa, según criterios de proximidad, los elementos de las representaciones. Los datos se agrupan sobre un espacio multidimensional, y muestran la organización de dichos elementos en el campo similar del grupo (Doise et al., 1993). Posteriormente, hizo un análisis de correspondencias múltiples. Este método se basa en el supuesto de que la similitud semántica de dos conceptos puede medirse según la distancia que los separa en el seno del mismo campo semántico y su distribución en el espacio dimensional.

Por otra parte, se utilizó un método de medida que posibilitó acceder a los principios que estructuran y dan coherencia a los campos de represen- tación (Di Giacomo, 1987; Doise et al., 1993; Cárdenas y Blanco, 2004). Tal método se constituye por la asociación libre de palabras a estímulos, lo cual permite elaborar diccionarios de asociación, que reflejan el campo semántico de cada palabra/ estímulo.

El diccionario de palabras fue creado a partir del total de las asociaciones a los estímulos presentados. La producción total de palabras ascendió a 9100 términos, lo que equivale a 20,92 vocablos por persona, y suponen un promedio de 3 términos por estímulo (para cada sujeto). Esta producción total de palabras se redujo a la cifra de 2656 términos, que se utilizaron como parte del diccionario definitivo. Para esta reducción se excluyeron términos semejantes y repetidos.

Para elaborar la matriz general de datos se consideró la presencia (valor de uno, 1) o la ausencia (valor de cero, 0) de términos del diccionario a cada estímulo. Por ejemplo: cada vez que un participante relacionaba los términos belleza, alegría, moda, madre o mujer al estímulo femenino se le asignaba el valor de uno (1). Dichos términos formaron el diccionario de asociaciones. Sobre esta matriz se aplicó la técnica del escalamiento multidimensional (MDS). Se hizo la matriz de distancia sobre medidas de similaridad para datos binarios; en ese caso se utilizó el coeficiente Phi (de cuatro puntos).

A continuación se presenta el MDS, el cual demuestra más claramente la posición donde se ubicaron los campos de significado compuesto por los estímulos planteados. El análisis obtuvo como base la exploración de la relación de estímulospalabras. Además, el análisis permitió llevar las distancias obtenidas a un espacio bidimensional. Las proximidades entre objetos son representadas como distancias entre puntos en un mapa (figura 1). Por medio de tal procedimiento se obtuvieron los ejes (atributos de los estímulos) que los participantes utilizaron para calcular las proximidades, y ello permitió una interpretación de los factores que subyacen a las relaciones establecidas. A mayor cercanía entre estímulos se encontrará una mayor valoración de parecidos. 
Figura 1. Asociación libre MDS, representación espacial para los siete estímulos

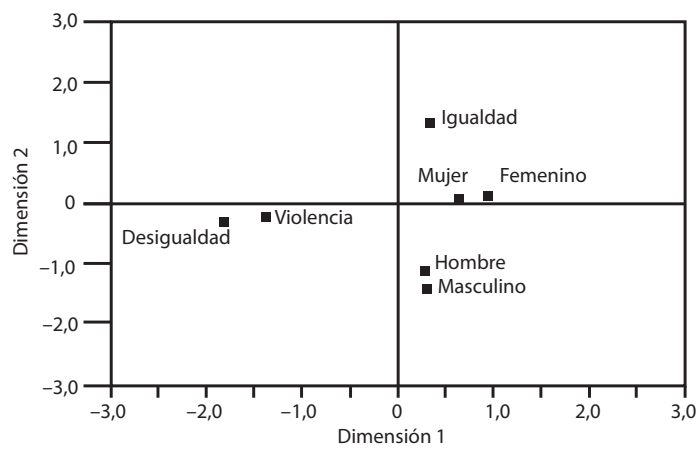

El eje uno opone las palabras Femenino y Mujer a Desigualdad, ubicadas en los extremos polares de dicho continuo; cerca de la palabra Desigualdad se encuentra la palabra Violencia. De este modo, la primera dimensión utilizada señala un eje que opone el fenómeno de la desigualdad/violencia a quienes podrían ser las probables afectadas de dicho proceso: las mujeres. Por otra parte, la primera dimensión podría estar indicando una percepción de la violencia contra las mujeres como un fenómeno universal basado en la desigualdad de género.

El segundo eje opone los estímulos Igualdad y Masculino. La palabra Masculino se vinculó a Hombre. La distancia entre los estímulos Igualdad y Masculino parece sugerir que los hombres están siendo evaluados por la muestra al margen de la idea de igualdad. De todas formas, parecen intuir que el concepto igualdad se aproxima más a los estímulos Mujer y Femenino, con los cuales se pone en relación en la primera dimensión, y ello denota, quizás, la idea de que el debate acerca de la igualdad fue (y, en cierta medida, todavía lo es) declarado y visibilizado por las mujeres.

En este punto cabe revisar los análisis de Correspondencias Múltiples más a menudo asociados a los estímulos utilizados en la Asociación Libre de Palabras. El Análisis Factorial de Correspondencias (AFC) es un método multivariante aplicable a variables categóricas, y cuyo objetivo es explorar las potenciales asociaciones entre variables cuali- tativas. Se puede interpretar dicho análisis como una radiografía que revela las estructuras presentes en la tabla de datos, pero ocultos a la limitada percepción del observador (Cornejo, 1998; Joaristi y Lizasoain, 2000). Se trata de la conexión o relación recíproca que existe entre dos elementos o conjuntos equivalentes. Tiene como objetivo profundizar en las relaciones de dependencia entre dos variables cualitativas, insistiendo en la explicación de cómo los valores de ambas se relacionan unos con otros.

Para los fines del presente trabajo, la atención se centró en lo que se ha denominado diferencial semántico de campos asociativos (Cornejo, 1998); es decir, una forma de Análisis de Correspondencias Múltiples que trabaja sobre material verbal proveniente de la asociación libre de palabras a una serie de estímulos.

Con el fin de obtener los resultados, se correlacionaron las palabras más indicadas para cada estímulo propuesto con el sexo de los participantes, a través del procedimiento de Análisis de Homogeneidad (Homals). La lógica del Análisis Factorial de Correspondencias (AFC), o Análisis de Homogeneidad (Homals) consiste en asignar determinadas coordenadas a individuos, categorías y variables en función de una serie de dimensiones que maximicen la diferencia entre ellas.

El Análisis Factorial de Correspondencias proporciona diversos parámetros que reflejan la medida en la que los factores calculados explican las diferencias entre las diferentes categorías, el grado en el que cada una queda recogida por los diferentes factores, y la contribución de cada una de las variables al factor; además, calcula las coordenadas en cada uno de los factores para los diferentes individuos, las categorías o las variables.

De este modo, las figuras entregan una representación de las proximidades entre las variables como distancias entre puntos en un espacio bidimensional. La observación de dichas proximidades o distancias permitió interpretar los factores que subyacen a las relaciones entabladas entre las variables. Los análisis que se presentan exploran las asociaciones hechas (campos semánticos) a los 
estímulos Femenino y Masculino, pues concentran las opiniones, las creencias y las posiciones más interesantes y provocativas al debate propuesto.

Figura 2. Análisis de correspondencias (Homals) Estímulo Femenino

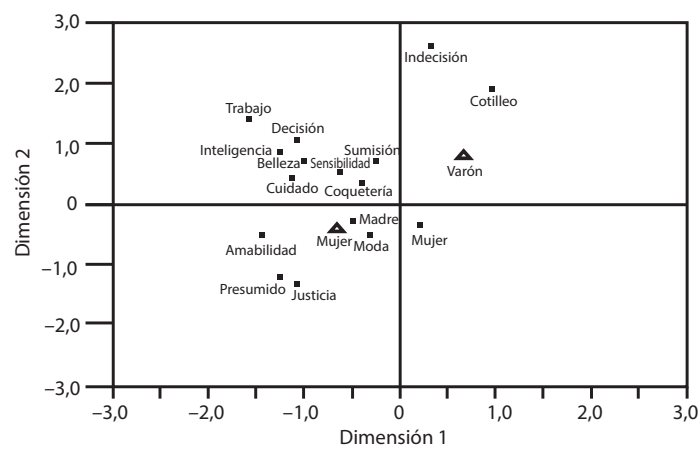

Los datos obtenidos informan acerca de los principales términos asociados al estímulo Femenino. En la dimensión 1 se encuentra la oposición de las palabras cotilleo y trabajo; la estructura de representación más cercana al segundo término, trabajo, es la formada por las palabras decisión, inteligencia, belleza, sensibilidad y cuidado. Por un lado, la representación de lo femenino, informada principalmente por las mujeres, está vinculada a características que expresan un carácter de decisión y acción. Se consideró que esta dimensión podría estar indicando una ruptura respecto a un referente fundamentalista que, por lo general, reserva dichas características al universo masculino. Por otro, lo femenino es representado por las palabras belleza, sensibilidad y cuidado, que también aparecen en ese campo. Aquí, las palabras indicadas estarían resaltando algunas características que reproducen el sistema de género. La primera podría estar indicando cuáles son las expectativas que definen lo femenino. En ese caso, tienen que ver con un patrón de belleza para ser mantenido. Las otras dos, que se asocian a ese campo de forma un poco más tardía, reproducen estereotipos de género, en la medida en que vinculan la imagen de lo femenino con las características de ternura y afectividad relacionadas con el cuidado y la preocupación por los demás.
Así pues, aunque las mujeres reconozcan que lo femenino engloba una serie de características potentes (decisión e inteligencia), todavía hablan de belleza, sensibilidad y cuidado como propias de la feminidad. Por un lado, las mujeres rescatan un punto "fuerte" de feminidad asociado a la independencia y la autonomía. Por otro, plasman un modelo sociocultural establecido y asimilado que enmarca lo femenino en el plano de lo estético y lo afectivo. Ese aspecto se ve reforzado por otro par de términos indicado por las mujeres: amabilidad y coquetería. Se estaría frente a una representación que, de algún modo, circula en el medio social y adscribe los contenidos considerados referentes a feminidad.

Según los hombres consultados, lo femenino sugiere cotilleo; es decir, lo femenino está vinculado al típico "comadreo", característica que reproduce una mentalidad estereotipada que encorseta lo femenino a un universo superficial y fútil, actuaciones no valoradas positivamente dentro del marco social, pues tienen un fondo bastante peyorativo. De este modo, la primera dimensión podría estar aludiendo a una cierta oposición frente a las características atribuidas a lo femenino. Sería una dimensión que resalta una feminidad al mismo tiempo dinámica, opuesta a una feminidad cargada de estereotipos. Fundamentalmente, se puede observar que se forma una representación bastante dicotomizada acerca de lo femenino.

En la dimensión 2 se encontró una oposición entre los términos indecisión, a justicia y presumida. El primer término fue más indicado por la muestra de hombres, y los dos últimos, por las mujeres. La palabra indecisión, vinculada al estímulo inductor (Femenino), sugiere que los hombres recrean un universo simbólico asociando lo femenino a características de duda e inseguridad. Ese dato es interesante, pues los hombres asocian al estímulo inductor justo lo contrario que a las mujeres, quienes, según hemos visto, asocian a dicho estímulo términos vinculados a decisión. Así, el debate de género se encuentra acordonado por un binomio (decisión $\times$ indecisión), cuyos pares, además de opuestos, son excluyentes. En la dimensión que ahora se interpreta, las mujeres indican los términos justicia y presumida, los cuales nada tienen 
que ver con los significados oriundos del término indecisión. Por justicia se entiende la posibilidad de acceso de las personas a la igualdad de oportunidades, el reconocimiento de la diversidad sin que esta signifique razón para la discriminación. Las mujeres indican imágenes y representaciones más ancladas en el campo sociopolítico buscando otros significados posibles para designar lo que es femenino.

En el epicentro del eje se observa la formación de un campo semántico con las palabras sumisión, moda, mujer y madre, términos indicados por ambos participantes. La palabra sumisión denota la relación que todavía existe entre lo femenino y los valores de subordinación y sometimiento. Las palabras mujer y madre, asociadas al estímulo femenino, quizás estén reflejando el trasfondo de los debates de género, los cuales sostienen que dentro del sistema social se producen y se reproducen expectativas y papeles asignados a los sexos en función de las características biológicas.

En términos generales, se puede ver cómo la representación social de la muestra acerca de lo femenino recae sobre ciertas creencias, extendidas en lo social, que lo asocian a lo reproductivo y a una serie de características que vienen dadas por la naturaleza. La palabra moda, asociada a lo femenino, lleva a pensar en las prácticas generalizadoras que homogenizan lo diverso bajo determinados patrones; sobre todo, los vinculados a la manutención de un perfil relacionado con la imagen ideal.

Figura 3. Análisis de correspondencias (Homals) Estímulo Masculino.

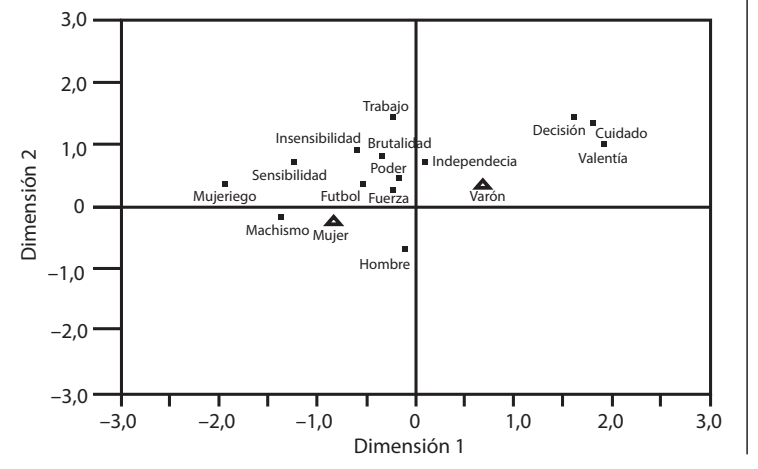

En esta parte aparecen los principales términos presentados por los participantes al estímulo inductor masculino. La dimensión 1 distribuye a lo largo del eje a los dos sexos; cada uno, asociado a un campo semántico. Por un lado, los hombres indican la palabra valentía. Las mujeres, por su parte, indican la palabra mujeriego. Cerca del término mujeriego se encuentran las palabras machismo y sensibilidad. Las palabras machismo y mujeriego revelan una connotación negativa a la hora de definir lo masculino, $y$, a la vez, revelan representaciones difundidas en el dominio social como propias de la masculinidad. Cuando utilizan la palabra sensibilidad parecen indicar otro modelo de lo masculino que admite una característica valorada positivamente, y que responde a un adjetivo vinculado de forma directa con las emociones. La palabra valentía, utilizada por los chicos, puede estar indicando la creencia de que lo masculino está directamente vinculado con la idea de coraje.

En el campo representacional de las mujeres se forma un núcleo bastante homogéneo, que alberga las palabras insensibilidad, fútbol y brutalidad. Tal bloque de términos parece corroborar el anterior, pues, una vez más, las mujeres eligen palabras negativas para representar lo masculino. La palabra fútbol parece indicar que las mujeres consideran esta actividad muy vinculada a lo masculino.

La dimensión 2 opone la palabras decisión, cuidado y trabajo a hombre. Decisión y cuidado, atributos elegidos por los hombres a la hora de representar lo masculino, resaltan una idea bastante positiva en el plano de la acción (decisión) y en el plano del afecto (cuidado). La palabra trabajo, situada en el polo izquierdo, revela, quizás, la idea de una representación social de lo masculino como productivo y proveedor.

En el epicentro del eje se ve la formación de un campo semántico con las palabras independencia, poder y fuerza. En términos generales, se aprecia que los vocablos utilizados en ese campo se encuentran vinculados a los modelos de masculinidad construidos como referentes. En ese sentido, el núcleo de la representación parece relacionar 
lo masculino con el ejercicio de la fuerza y la detentación del poder. La palabra independencia remite a un estereotipo de género que vincula lo masculino a la condición de autonomía y libertad.

\section{Discusión}

En términos generales, se puede decir que la TRS se articula tanto con la vida colectiva de una sociedad como con los procesos de constitución simbólica utilizados por las personas para dar sentido al mundo, entenderlo y en él hallar el propio lugar, a través de una identidad social. De este modo, es evidente cómo las RS, entendidas en su calidad de fenómeno psicosocial, están necesariamente radicadas en el espacio público y en los procesos a través de los cuales el ser humano desarrolla una identidad, crea símbolos y se abre a la diversidad de otros. El espacio público, como un lugar de la alteridad, fornece a las RS de un terreno donde ellas pueden ser cultivadas y establecidas (Guareschi y Jovchelovich, 1995).

Las RS emergen como un fenómeno pegado al tejido social. Lo social, en términos generales, asume las condiciones concretas de la vida, envuelve desde relaciones sociales de producción hasta mecanismos institucionales de diversos órdenes. Es importante destacar que, a través de la acción de sujetos sociales actuando en el espacio común de la esfera pública, surge algo así como un lugar donde una comunidad puede desarrollar y sostener saberes sobre sí misma; en otras palabras, elaborar y fijar representaciones sociales.

En esta investigación se pudo considerar la información aportada por las personas participantes como una lectura o una interpretación sobre la realidad que intentan comprender. Sin duda, el acto de interpretación de la realidad se da en aquellos procesos de comunicación, asentada en la mediación entre un discurso construido de modo colectivo que acaba generando RS.

La necesidad de interpretar las RS de género podría suscitar otras cuestiones con nuevas perspectivas, y así redefinir en nuevos términos los antiguos cuestionamientos. Además, los hallazgos encontrados abren posibilidades para la reflexión acerca de las estrategias políticas actuales y los planteamientos futuros. Desde el punto de vista del presente trabajo, se hace necesario entender cómo los participantes representan la categoría género; cómo se sirven de ella para comunicar las reglas de las relaciones sociales; también, contrastar las diferencias percibidas entre hombres y mujeres acerca de los indicadores de género ( $\mathrm{pa}$ pel de género, estereotipo de género, masculinidad y feminidad).

Es importante resaltar que las interpretaciones se refieren a los campos semánticos en torno a los estímulos Femenino y Masculino, de los cuales se analizará el núcleo de RS, y, luego, las RS más periféricas respecto a cada estímulo. Se puede considerar que la información atribuida por los jóvenes es una lectura o una interpretación sobre el contexto que intentan comprender. Sabemos que la interpretación de la realidad se asienta, generalmente, sobre un discurso construido de modo colectivo, tomando como base RS anteriores. Generalmente, dicha interpretación se cruza con las distintas pertenencias y adscripciones que una persona posee; en particular, por las palabras-estímulos que más se destacaron a la hora de hacer la Asociación Libre de Palabras.

Primeramente, se analizó el núcleo de las RS formado en torno a cada estímulo capturando los elementos y las características particulares según hombres y mujeres (muestra total). Se entiende que la interpretación de determinada realidad se hace siempre a partir de ciertas dimensiones más estables; por consiguiente, las fluctuaciones serían graduales, no afectarían el marco más amplio o la esencia más profunda desde donde se llevan a cabo estas operaciones interpretativas.

El análisis se despliega examinando primero los núcleos de RS, y luego, los campos semánticos más periféricos, según los hombres y las mujeres por separado (muestra segmentada). Las RS más periféricas (incluso, las interpretaciones novedosas acerca de un fenómeno nuevo) pueden alterar los elementos más profundos, si bien se debe reconocer una tendencia a que sean utilizadas para confirmar el núcleo más estable de la RS. Como 
se verá, no obstante se trate de atribuciones novedosas que servirían para contradecir las RS más arraigadas, suelen conformar el núcleo de la RS.

En relación con el campo semántico elaborado por mujeres y hombres (epicentro del eje) en torno al concepto Femenino, se puede afirmar que se encuentra anclado en estereotipos sexistas profundamente enraizados en lo social. Las palabras que gravitan en torno al eje central hablan, primero, de las características biológicas adscritas al femenino: ser mujer, y, por consiguiente, ser madre. Aun cuando innúmeras investigaciones muestran que el concepto de sexo no es válido para explicar las diferencias entre hombres y mujeres en las más variadas culturas, en el caso específico de este trabajo se verá que los adolescentes consultados no diferencian lo biológico, lo atribuido al sexo, de lo cultural, determinado por el género.

Como afirma Pateman, (1996), "la posición de la mujer no está dictada por la naturaleza, por la biología o por el sexo, sino que es una cuestión que depende de un artificio político y social" (p. 308). Al contrario del sexo, el género tiene un carácter sociocultural, es una construcción simbólica y discursiva que reúne los elementos, las adscripciones y las prácticas que cada sociedad considera propios de lo masculino y lo femenino.

Además de lo anterior, las diferencias entre los géneros, asociadas a las distinciones biológicas, han servido para acreditar diferentes posibilidades y lugares sociales que serían considerados propios de cada género. Así, la afirmación de que hombres y mujeres son diferentes pauta la idea de la existencia de una esencia masculina y una femenina, lo que muchas veces justifica las relaciones desiguales entre los géneros.

El par de palabras sumisión y moda completa el campo semántico. El término moda indica modelos normativos que imponen a los cuerpos de las mujeres un "deber de buena apariencia", lo cual presupone mantenerse acorde con los cánones de belleza y juventud. La búsqueda por los signos identificatorios de feminidad acaba produciendo una obsesión en dirección a la imagen ideal en un número significativo de mujeres (Sant’Anna, 1995).
En lo relacionado con el estímulo Femenino, este también es caracterizado como sumiso, una mujer que no goza de autonomía para sí misma, algo que denota una desigual distribución del ejercicio de poder entre los sexos. La posición de un género respecto al otro es un eje crucial por donde discurren las asimetrías de poder en lo social. A lo largo de la historia se ha legitimado una creencia de la inferioridad de la mujer respecto al hombre.

De forma parcial, lo femenino es caracterizado por las mujeres como: decisión, inteligencia, justicia, belleza, coquetería, presumida, sensibilidad, cuidado, y amabilidad, y por los hombres, como cotilleo e indecisión. Lo primero que salta a la vista es el contraste en la formulación de los campos semánticos. Las mujeres adscriben al femenino características más positivas que los hombres; sin embargo, siguen repitiendo características y prácticas ya presentadas en el núcleo de la RS, vinculadas a la idea tradicional acerca de lo femenino: ser bella, coqueta, presumida, sensible, cuidadora y amable. Tales estereotipos positivos responden a modelos de mujeres "buenas", cuya forma de ser y de hacer está en función de los deseos y las necesidades de las demás personas.

Por otro lado, las mujeres destacan características novedosas respecto al núcleo de RS de lo femenino. Las palabras decisión, inteligencia y justicia hablan de un femenino alejado del estereotipo y que va ganando terreno social. Las palabras decisión y justicia, por ejemplo, indican la necesidad de participación de las mujeres en diferentes esferas (económica, política, social, familiar, toma de decisiones etc.) sobre bases participativas e igualdad de oportunidades. Pese a que son minoritarios en la RS de lo femenino, dichos términos remiten a pensar en los cambios sensibles, tanto en aspectos subjetivos como en relación con el sitio que tradicionalmente la sociedad ha designado a la mujer, y que van cambiando poco a poco y siendo introducidos en los debates públicos.

El campo semántico correspondiente a los hombres atribuye a lo femenino el cotilleo y la indecisión. En conjunto, son características que ofrecen un "yo débil" y denotan una desvalorización hacia 
las mujeres, pues los campos semánticos formulados acerca de lo femenino albergan palabras que asumen una tendencia estereotipada. Como acabamos de analizar, la estructura representacional es aludida por medio de las palabras sumisión, moda, mujer y madre. Se ve aquí cómo el juicio se da de forma cualitativa y más libre (Asociación Libre de Palabras), y la producción de los campos de significados se vincula visiblemente a la representación de lo femenino, centrada, a su vez, en características asociadas a la naturaleza, la emoción y el cuidado.

Por otro lado, se analizó el campo semántico formado en torno al estímulo Masculino. El núcleo de la RS presenta un masculino vinculado al trabajo, a la independencia, al poder y a la fuerza. Se encuentra una imagen que resalta, ante todo, el carácter activo, atrevido y valiente, características reconocidas y valoradas socialmente. Las atribuciones se caracterizan por el énfasis en la virilidad, la fuerza y el trabajo, las cuales contribuyen a legitimar la creencia en la posición superior del hombre sobre la mujer, y su predominio en valores y jerarquías en los procesos de socialización de la cultura.

La construcción de un orden simbólico basado en la pretensa superioridad masculina implica una serie de comportamientos y actitudes estereotipadas que conducen la subordinación de un sexo a otro. Ello se corresponde con el hecho, de validez prácticamente universal, de que los hombres se dedican más a las tareas extradomésticas, que comprenden desde el ámbito económico y político hasta el cultural (Amorós, 1995). Dichas tareas otorgan al hombre un lugar diferenciado en las relaciones. Por otro lado, la fuerza suele ser un atributo comúnmente vinculado a lo masculino; incluso, determinado como una característica biológica que confiere superioridad.

La RS parcial acerca de lo masculino es variable respecto a lo que piensan las mujeres y los hombres. Para las mujeres lo masculino se vincula a mujeriego, machista, brutalidad, insensible-sensible y fútbol. Aunque se trate de una RS parcial (lo masculino visto por las chicas), es notorio cómo se hace presente una esencia que repite características nucleares respecto al estímulo en cuestión. Una vez más, se evidencia la perspectiva pública de lo masculino (mujeriego, machista, fútbol).

Llama la atención la dicotomía sensible-insensible, que cuestiona la construcción cultural y simbólica acerca de la afirmación sexista de que lo masculino está directamente asociado a la insensibilidad. Por otro lado, los hombres dicen que lo masculino está relacionado con valentía, decisión y cuidado. Por un lado, el hecho de que los hombres relacionen a lo masculino la valentía y la decisión implica dificultades para cuestionar un papel que encasilla a los hombres y confirma creencias provenientes de los procesos de socialización. Por otro, si el adjetivo cuidado se refiere a protección a las mujeres indicaría formas desiguales de relación, y si dicho adjetivo estuviese vinculado a la atención de las necesidades fisiológicas y afectivas de ambos, se podría vislumbrar un cuestionamiento de papeles por parte de los hombres.

Los resultados obtenidos en la exploración de las dimensiones subjetivas, y alusivos a un complejo campo de estudio, deben ser necesariamente contextualizados dentro del marco histórico y cultural donde han ocurrido. Delinean, en cualquier caso, indicaciones como sugerencias de avance para futuras investigaciones dentro de este ámbito, enfocadas, en mayor medida, en el desarrollo de conceptos, tanto de estrategias metodológicas como de resultados que abarquen los fenómenos desarrollados en un plano colectivo.

Una reflexión depurada acerca de los hallazgos del presente trabajo permite constatar la importancia de investigar con mayor detenimiento otros temas importantes, acordes con los estudios de género: los aspectos endogrupales implicados en el sentimiento de identificación y las RS de género, incluyendo métodos cualitativos, por cuanto estos otorgan la posibilidad de complementar la riqueza de los datos a la hora de acceder a las RS; por tanto, sirven como herramienta de análisis de los procesos que se quiere estudiar. 


\section{Referencias}

Amorós, C. (1990). Violencia contra las mujeres y pactos patriarcales. En Maquieira, V., \& Sánchez, C. (Ed.) Violencia y sociedad patriarcal (pp. 1-15). Madrid: Pablo Iglesias.

Amorós, C. (1995). 10 Palabras clave sobre mujer. Navarra: Verbo Divino.

Asch, S. (1962). Psicología social. Buenos Aires: Eudeba.

Banchs, M. (1986). Concepto de representaciones sociales: análisis comparativo. Revista costarricense de psicología, 89, 27-40.

Blanco, A. (1996). Vygotsky, Lewin y Mead: los fundamentos clásicos de la psicología social. En Páez, D., Blanco, A. (Eds.) La teoría sociocultural y la psicología social actual. Madrid: Fundación Infancia y Aprendizaje.

Blanco, A. (1988). Cinco tradiciones en la Psicología social. Madrid: Morata.

Cárdenas, M. y Blanco, A. (2004). Las representaciones sociales del movimiento antiglobalización. Revista de psicología política, 28, 27-54

Cárdenas Castro, M. (2005). Psicosociología de los nuevos movimientos sociales: El movimiento antiglobalización (representación, conflicto $e$ influencia social). (Tesis director, Universidad Autónoma de Madrid, Madrid, España). Recuperado de https://repositorio.uam.es/ handle/10486/8898

Cornejo, J. M. (1998). Técnicas de investigación social. El análisis de correspondencias. Teoría y práctica. Barcelona: Promociones y Publicaciones Universitarias.

Di Giacomo, J. P. (1980). Intergroup alliances and rejections within a protest movement (analysis of the social representations)". European Journal of Social Psychology, 10(4), 329-344.

Di Giacomo, J. P. (1987). Teoría y método de análisis de las representaciones sociales. En D. Páez. Pensamiento, individuo y sociedad.
Cognición y representaciones. Madrid: Fudamentos (278-295).

Doise, W., Clémence, A., \& Lorenzi-Cioldi, F. (1993). The quantitative analysis of social representations. Hemel Hempstead: HarvesterWheatsheaf.

Freire, P. (1999). Pedagogia da autonomia: Saberes necessários à prática educativa. São Paulo: Paz e Terra.

Guareschi, P. A., \& Jovchelovitch, S. (Orgs.) (1995). Textos em representações sociais. Rio de Janeiro: Vozes.

Joaristi Olariaga, L., \& Lizasoain Hernández, L. (2000) Análisis de correspondencias. Cuaderno de estadística 5. Madrid: La MurallaHespérides.

Jodelet, D. (1985). La representación social: fenómenos, conceptos y teorías. En Moscovici, S. Psicología Social (Vol. 2.). Barcelona: Paidós.

Jodelet, D. (1984). The representation of the body and its transformations. En Farr, R. M., \& Moscovici, S. (Eds.). Social Representations. Cambridge: Cambridge University Press.

Jovchelovitch, S. (2007). Knowledge in Context: representations, community and culture. Londres: Routledge.

Lorente Acosta, M. A. (2001). Mi marido me pega lo normal. Agresión a la mujer; realidades y mitos. Barcelona: Ares y Mares.

Moscovici, S. (1988). Notes towards a description of social representation. European Journal of Social Psychology, 18, 211-250.

Moscovici, S. (1987). Social collectivities. En Hulse, M. (Ed.) Essays in Honor of Elias Canetti (pp. 42-59). Londres: André Deutsch.

Moscovici, S. (1979). El psicoanálisis, su imagen y su público. Buenos Aires: Huemul.

Moscovici, S. (1973). Foreword. I Herzlich, C. Health and illness: a social psychological analysis. Londres: Academic Press. 
Moscovici, S. (1981). Psicología de las minorías activas. Madrid: Morata.

Moscovici, S. (2003). Representações sociais: investigações em psicologia social. Rio de Janeiro: Vozes.

Moscovici, S. (1976). Social influence and social change. Londres: Academic Press.

Pateman, C. (1996). Críticas feministas a la dicotomía publico/privado. En Castells, C. Perspectivas feministas en teoría política (pp. xx$\mathrm{xx}$ ). Barcelona: Paidós.

Pérez, J. A., Moscovici, S., \& Chulvi, B. (2002). Natura o cultura como principio de clasificación social. Anclaje de representaciones sociales sobre minorías étnicas. Revista de Psicología Social, 17(1), 51-67.
Sanmartín, J. (2002). Violencia contra las mujeres: causas y efectos. En San Martín, J. Actas de la II Conferencia Internacional sobre Violencia Contra las Mujeres. Madrid: Ayuntamiento de Madrid.

Sant'Anna, D. B. (1995). Políticas do corpo. São Paulo: Estação Liberdade.

Schutz, A. (1982). Commonsense and scientific interpretations of human action. En Collected Papers I. Hague: Martinus Nijhoff.

Velázquez, S. (2003). Violencias cotidianas, violencia de género: escuchar, comprender, ayudar. Buenos Aires: Paidós. 